\title{
Evaluation of frequency and type of errors detected by a computerized record and verify system during radiation treatment
}

\author{
Nicole Barthelemy-Brichant ${ }^{\mathrm{a}, *}$, Jacques Sabatier ${ }^{\mathrm{a}}$, Walthère Dewé ${ }^{\mathrm{b}}$, Adelin Albert ${ }^{\mathrm{b}}$, \\ Jean-Marie Deneufbourg ${ }^{\mathrm{a}}$ \\ ${ }^{a}$ Department of Radiotherapy, Centre Hospitalier Universitaire de Liège, Campus du Sart Tilman, B 35, B-4000 Liège, Belgium \\ ${ }^{\mathrm{b}}$ Department of Medical Informatics and Statistics, Centre Hospitalier Universitaire de Liège, Campus du Sart Tilman, B 35, B-4000 Liege, Belgium
}

Received 26 February 1999; received in revised form 6 August 1999; accepted 22 September 1999

\begin{abstract}
Background: Computerized record and verify systems (RVS) have been introduced to improve the precision of radiation treatment delivery. These systems prevent the delivery of ionizing radiations when the settings of the treatment machine do not match the intended parameters within some maximal authorized deviation.

Purpose: To assess the potential alteration of the frequency of errors associated with the use of RVS during radiation treatment delivery.

Materials and methods: The software of the RVS was altered in order to record the settings actually used for radiation treatment delivery whereas the verification function was suppressed. At the end of the study period, the settings used during daily administration of radiation treatment were compared to the parameters recorded in the RVS using the computer. They were also compared with the planned ones written in the patient treatment chart.

Results: Out of the 147476 parameters examined during the study period, $678(0.46 \%)$ were set erroneously. At least one error occurred in $628(3.22 \%)$ of the 19512 treated fields. An erroneous parameter was introduced in the RVS memory in $22(1.17 \%)$ of the 1885 fields.

Conclusions: RVS has the potential to improve precision of radiation treatment delivery by detecting a significant number of setting errors. However, excessive confidence in RVS could lead to repeated errors as there is a potential for the entry of erroneous parameters into the RVS memory. (C) 1999 Elsevier Science Ireland Ltd. All rights reserved.
\end{abstract}

Keywords: Radiation therapy; Errors; Quality control; Record and verify system

\section{Introduction}

In cancer patients, the goal of radiotherapy is to yield the greatest possible uncomplicated rate of local and regional control. In this respect, the outcome of radiation therapy clearly depends on the precision with which the dose is delivered to the target volume. Dose variations of $10 \%$ either way can significantly change the risk of side effects or the probability of tumor control. Therefore, it is recommended that the uncertainty associated with dose distribution should be less than 5\% [1].

In any radiation treatment, several steps are involved. These can be divided into three main parts: treatment prescription, treatment preparation and treatment delivery. Due to the numerous steps and the large group of persons involved in the preparation and the execution of radiation treatments, the transfer of information from one step to the next is critical as it may lead to errors. In addition, because

\footnotetext{
* Corresponding author.
}

the delivery of external beam radiation is a complex process involving the setting of at least 15 parameters for each field, errors that can potentially alter dose distribution and hence the outcome of the treatment are likely to occur during daily treatment delivery [11].

Quality-assurance programs have been developed to improve the precision and accuracy of radiation treatments. They include, among others, periodic verification of the simulators, the treatment units and dosimetry. Improvement of radiation treatments is also the aim of the use of verification films, immobilization devices, in vivo dosimetry and record and verify systems (RVS) [13].

Computer-assisted RVS aim at reducing the setting errors during daily radiation treatment delivery. However, the actual impact of RVS on the error frequency occurring during radiation treatment delivery under normal workload conditions has scarcely been investigated. The present open blind study was designed (i) to estimate the frequency of errors in treatment settings, and (ii) to evaluate the potential benefits resulting from the use of a computer assisted RVS. 


\section{Materials and methods}

This study, approved by the Institutional Ethics Committee, included most treatments performed during 6 consecutive months in the Department of Radiotherapy of the Liege University Hospital.

\subsection{Staff and equipment}

Radiation treatments are prescribed by the radiation oncologists. Treatment preparation is performed in close collaboration by the radiation oncologists, the physicists and the dosimetrists. The treatment parameters are introduced in the RVS computer as well as in the patient treatment chart by these staff members. Radiation nurses or radiographers carry out the daily treatment settings as well as the irradiation according to the settings written in the patient chart.

The treatment facility is equipped with a simulator (Simulix, Oldelft) and three treatment machines: one Cobalt unit (Alcyon 2, CGR MeV) and two linacs. One of the linacs (ORION 6, CGR MeV) produces a $6 \mathrm{MV}$ photon beam whereas the other one (Saturne $2+, \mathrm{CCR} \mathrm{MeV}$ ) is able to deliver multiple energy electrons beams or dual energy photons. Asymmetrical beams are available on the two linacs.

The three treatment machines differ by the display of some of their settings. On the Alcyon, the field length and width, the gantry angle as well as the collimator rotation are displayed analogically. On the two linacs, these settings are displayed digitally as well as any other setting on the three machines.

\subsection{Record and verify system}

The treatment machines and the simulator are interfaced and connected by Ethernet network to a centralized RVS (Sincer, CGR MeV) consisting of four Vaxstations.

Different treatment parameters are controlled by the RVS. They include geometrical, prescription as well as dosimetry parameters. Some treatment settings can be transferred automatically from the simulator to the RVS, whereas others have to be introduced manually via the keyboard (Table 1). On each machine the couch settings are not controlled by the RVS nor is the use of a blocking tray on the Alcyon. Six parameters per field were controlled on the Alcyon, compared to seven and nine parameters on the Orion and the Saturne, respectively (Table 1).

Daily treatment setting is performed according to the parameters written in the patient chart. When the radiation nurses or the radiographers attempt to turn the beam on, the treatment machine transmits the parameter settings to the RVS. If the value of at least one parameter differs from the one recorded in the RVS memory by more than a specified tolerance limit, the radiation beam is inhibited.
Table 1

Parameters entered manually into the record and verify system (RVS) and controlled in the present study according to equipment

\begin{tabular}{|c|c|c|c|}
\hline \multirow[t]{2}{*}{ Parameter } & \multicolumn{3}{|c|}{ Treatment machine } \\
\hline & Alcyon & Orion & Saturne \\
\hline \multicolumn{4}{|l|}{ Geometry } \\
\hline Field size $X^{\mathrm{a}}$ & Yes & Yes & Yes \\
\hline Field size $Y^{\mathrm{a}}$ & Yes & Yes & Yes \\
\hline Collimator angle $^{\mathrm{a}}$ & Yes & Yes & Yes \\
\hline Gantry angle $^{\mathrm{a}}$ & Yes & Yes & Yes \\
\hline Source skin distance & No & No & No \\
\hline Applicator & No & No & Yes \\
\hline Start and end angle in arctherapy & No & No & No \\
\hline Wedge or blocking tray number & Yes & Yes & Yes \\
\hline Asymmetry & No & Yes & Yes \\
\hline \multicolumn{4}{|l|}{ Prescription } \\
\hline Treatment machine & No & No & No \\
\hline Energy & No & No & Yes \\
\hline Dose per field & No & No & No \\
\hline Treatment schedule & No & No & No \\
\hline \multicolumn{4}{|l|}{ Dosimetry } \\
\hline Field dose at the maximum & No & No & No \\
\hline Monitor units or treatment time & Yes & Yes & Yes \\
\hline
\end{tabular}

${ }^{\mathrm{a}}$ Can also be entered automatically in the RVS from the simulator.

\subsection{Study design}

For the purpose of the study, the software of the RVS was altered to suppress the verification function while maintaining the recording of the settings actually used during the treatment sessions. In practice, the radiation nurses or the radiographers set the treatment machine according to the patient chart. Then, the patient's name as well as the identification number of the field to be treated were introduced into the RVS computer via the keyboard. Afterwards, the screen was automatically switched off and the beam was turned on.

When the 6-month study period had elapsed, the settings actually used during daily administration of radiation treatment were compared to those recorded in the RVS using the computer. They were also compared with the planned instructions written in the patient chart.

\subsection{Definition of errors}

Discrepancies between the actually used settings and those recorded in the RVS were considered as errors. These errors were termed as 'false' errors when the settings used during treatment delivery were different from those introduced in the RVS but similar to the planned ones as appearing in the data written in the patient chart. Any discrepancy between actually used settings and those written in both the patient chart and the RVS memory were considered 
as 'true' errors. True errors were further categorized into minor or major ones. Minor errors were defined as not affecting treatment outcome. By contrast, major errors were potentially harmful to the patient. Criteria for distinguishing between minor and major errors are based on the RVS tolerance table used in our daily clinical practice (Table 2). Indeed, in daily clinical work, some flexibility must be allowed for machine parameters to avoid an excess of verification failures. A balance exists between loosening tolerance levels for the purpose of limiting error signals to a certain frequency and tightening them to maintain sufficient quality standards. Deviations in treatment time were expressed in seconds and in percentage of variation from the prescription. It is also worth mentioning that large collimator angle deviations are allowed during treatment with electrons beams as this is accepted for fields which have limits outlined on the skin. For this reason, any deviation beyond $10^{\circ}$ was considered as a minor deviation. The total number of true errors is the sum of minor and major errors.

\subsection{Statistical methods}

Error frequencies are reported as actual counts as well as percentages of the total number of treated fields or studied parameters. Error frequencies were compared by the classical chi-square test. Results were considered to be significant at the $5 \%$ critical level $(P<0.05)$. All calculations were carried out using SAS statistical package (version 6.12) (SAS Institute, Cary, NC).

\section{Results}

The study material consisted of the treatment sessions of 539 cancer patients treated by external radiation beam. There were 1885 different fields treated in 19512 sessions amounting to the settings of 147476 parameters.

\subsection{True errors}

The distribution of the number of true errors per field according to radiation equipment is given in Table 3 . The overall treatment settings were similar to the planned ones in $96.8 \%$ of the fields, whereas one or more deviations occurred in $3.22 \%$ of the cases. The distribution varied according to the radiation equipment in the sense that a significantly higher frequency of erroneous fields were treated with the Alcyon than with the Orion and the Saturne $(P<0.005)$.

We found that, out of the 147476 examined parameters, $678(0.46 \%)$ were set erroneously, among which 503 $(0.34 \%)$ presented a major deviation. Tables 4 and 5 display the total number of errors and the number of major errors, respectively for the different parameters and radiation equipments. Highly significant differences in error frequencies (both total and major) were found between the three equipments for all parameters. The overall proportion of true errors was higher when irradiation was delivered with the beam of the Alcyon $(4.39 \%)$ than when the Orion $(2.72 \%)$ or the Saturne $(3.35 \%)$ was used, despite a fewer number of parameters taken into account. As far as major errors were concerned, the corresponding figures were $2.81 \%$ (Alcyon) against $2.23 \%$ (Orion) and $2.65 \%$ (Saturne).

The gantry angle was set erroneously less frequently when the Orion was utilized than when using either the Saturne or the Alcyon $(P<0.001)$. The frequency of errors for the gantry was significantly higher $(P<0.001)$ when the position to be set was different from $0,90,180$ or $270^{\circ}$. In our study, $14912(76.4 \%)$ treated fields were set with these gantry angles, while in $4600(23.6 \%)$ fields other angles were used. In the former case, $13(0.08 \%)$ errors occurred (two minor and 11 major) and in the latter $34(0.74 \%)$ errors were detected (eight minor and 26 major). For both minor and major errors, differences in frequency were highly significant $(P<0.001)$.

Significantly more errors in collimator rotation occurred when the Alcyon was used $(P<0.001)$. A total of 15645 treated fields were set with a collimator rotation of 0,90 , 180 and $270^{\circ}$ and 3867 with other angles. For the classical angles, $45(0.29 \%)$ errors occurred (17 minor and 28 major) whereas for the others, $28(0.72 \%)$ errors were observed (eight minor and 20 major). Again, frequency differences for both minor and major errors were highly significant $(P<0.001)$.

Table 2

Definition of minor and major errors

\begin{tabular}{lll}
\hline Parameter & Minor error & Major error \\
\hline Gantry angle & $>2^{\circ}$ & $>5^{\circ}$ \\
Collimator rotation & $>2^{\circ} ;>10^{\circ}$ electron beam & $>5^{\circ}$ photon beam \\
Field size $(Y)$ & $>3 \mathrm{~mm}$ & $>5 \mathrm{~mm}$ \\
Field size $(X)$ & $>3 \mathrm{~mm}$ & $>5 \mathrm{~mm}$ \\
MU $^{\mathrm{a}}$ treatment time & Any variation below major error & $>30 \mathrm{MU}^{\mathrm{a}}$ or $>30 \mathrm{~s} ;>5 \%$ \\
Wedge, blocking trays & & Any difference \\
Applicator & & Any difference \\
Energy & Any difference \\
Asymmetry & Any difference \\
\hline
\end{tabular}

${ }^{\mathrm{a}} \mathrm{MU}$, Monitor unit. 
Table 3

Distribution of true errors per field (see text for definition) according to treatment machines ${ }^{\mathrm{a}}$

\begin{tabular}{|c|c|c|c|c|}
\hline Number of errors per field & Alcyon $(n=5698)^{*}$ & Orion $(n=5519)^{*}$ & Saturne $(n=8295)^{*}$ & Total $(n=19512)^{*}$ \\
\hline 0 & $5472(96.0)$ & $5373(97.4)$ & 8039 (96.9) & $18884(96.8)$ \\
\hline \multirow[t]{2}{*}{1} & $203(3.56)$ & $142(2.57)$ & $240(2.90)$ & $585(2.99)$ \\
\hline & $(68 / 135)$ & $(25 / 117)$ & $(55 / 185)$ & $(148 / 437)$ \\
\hline \multirow[t]{2}{*}{2} & $22(0.39)$ & $4(0.07)$ & $13(0.16)$ & $39(0.20)$ \\
\hline & $(11 / 11)$ & $(1 / 3)$ & $(0 / 13)$ & $(12 / 27)$ \\
\hline \multirow[t]{2}{*}{3} & $1(0.02)$ & $0(0)$ & $1(0.01)$ & $2(0.01)$ \\
\hline & $(0 / 1)$ & & $(1 / 0)$ & $(1 / 1)$ \\
\hline \multirow[t]{2}{*}{4} & 0 & 0 & $1(0.01)$ & $1(0.01)$ \\
\hline & & & $(0 / 1)$ & $(0 / 1)$ \\
\hline \multirow[t]{2}{*}{5} & 0 & 0 & $1(0.01)$ & $1(0.01)$ \\
\hline & & & $(0 / 1)$ & $(0 / 1)$ \\
\hline
\end{tabular}

${ }^{\mathrm{a}}$ Results are expressed as number $(\%)$ of treated fields per equipment. Minor/major errors are given in italics. $* n$, Number of treated fields.

There were 254 field sizes $X$ or $Y$ set erroneously, including 171 major deviations. Errors in field size occurred more frequently when the Alcyon was used $(P<0.001)$.

Deviations in treatment time occurred more frequently on the Saturne: $68(0.82 \%)$ vs. $22(0.39 \%)$ and $28(0.51 \%)$ on the Alcyon and the Orion, respectively. In terms of percent of erroneous time delivery, there was a higher level of major errors with Saturne beam. However, no difference was observed in the frequency of major deviations between the three machines when the variation in the treatment time was expressed in seconds or UM $(7(0.12 \%), 10(0.18 \%)$, $16(0.19 \%)$ on the Alcyon, Orion and Saturne, respectively).

Among the 13814 fields treated with Orion and Saturne, erroneous wedges or blocking trays were utilized in $0.95 \%$ of the sessions. A wrong asymmetrical beam was used in $0.19 \%$ of the sessions delivered by the Orion or the Saturne. A wrong energy was used in $0.3 \%$ of the Saturne treatment sessions.

We found a significantly higher frequency of errors during the first 6 weeks of the study period but no difference was observed between the days of the week nor the hours of the day (Fig. 1). A higher frequency of erroneous treatment settings was found when the treatment was delivered by recently certified radiation nurses or radiographers as compared to more experienced staff ( 385 out of 9406 parameters and 293 out of 10106 parameters, respectively $P<0.0001)$.

\subsection{False errors}

An erroneous parameter was introduced in the RVS memory in 22 of the 1885 fields (1.17\%), specifically in 216 of the 19512 treated fields $(1.1 \%)$ (Table 6).

\section{Discussion}

There is a potential risk for error settings during radiation therapy. Errors can occur at each step, from the prescription to the delivery of the radiation treatment. Quality control programs are designed to decrease the frequency of such errors and consequently to increase the precision of radiation treatments. A better accuracy should improve the efficacy and reduce the side effects of radiation therapy. Record and verifiy systems have been introduced to reduce the frequency of errors in parameter settings during daily treatment delivery. They also provide a complete and reliable record of treatment, not only useful to the clinical radio-

Table 4

Frequency of true errors (see text for definition) according to parameters and treatment machines ${ }^{\mathrm{a}}$

\begin{tabular}{|c|c|c|c|c|c|}
\hline Parameter & Alcyon $(n=5698)^{*}$ & Orion $(n=5519)^{*}$ & Saturne $(n=8295) *$ & Total $(n=19512)^{*}$ & $P$-value \\
\hline Gantry & $22(0.39)$ & $2(0.04)$ & $23(0.28)$ & $47(0.24)$ & $<0.001$ \\
\hline Collimator rotation & $36(0.63)$ & $11(0.2)$ & $26\left(\begin{array}{ll}0.3 & 1\end{array}\right)$ & $73(0,38)$ & $<0.001$ \\
\hline Field size $X$ & $68(1.20)$ & $21(0.38)$ & $17(0.20)$ & $106(0.54)$ & $<0.001$ \\
\hline Field sizeY & $101(1.77)$ & $16(0.29)$ & $31(0.37)$ & $148(0.77)$ & $<0.001$ \\
\hline Treatment time & $22(0.39)$ & $28\left(\begin{array}{lll}0.5 & 1\end{array}\right)$ & $68(0.82)$ & $118(0.6)$ & 0.003 \\
\hline Wedge & $1(0.01)$ & $59(1.06)$ & $73(0.88)$ & $133(0.68)$ & $<0.001$ \\
\hline Applicator & - & - & $1(0.01)$ & 1 & \\
\hline Energy & - & - & $26(0.31)$ & $26(0.13)$ & \\
\hline Asymmetry & - & $13(0.24)$ & $13(0.15)$ & $26(0.13)$ & 0.002 \\
\hline Total & $250(4.39)$ & $150(2.72)$ & $278(3.35)$ & $678(3.47)$ & \\
\hline
\end{tabular}

\footnotetext{
${ }^{\mathrm{a}}$ Results are expressed in terms of number (\%) of treated fields with erroneous parameters over total number of treated fields. Comparison of error frequencies between treatment machines $(P$-value). $* n$, Number of treated fields.
} 
Table 5

Frequency of major errors (see text for definition) according to parameters and treatment machines ${ }^{\mathrm{a}}$

\begin{tabular}{|c|c|c|c|c|c|}
\hline Parameter & Alcyon $(n=5698)^{*}$ & Orion $(n=5519) *$ & Saturne $(n=8295)^{*}$ & Total $(n=19512)^{*}$ & $P$-value \\
\hline Gantry & $15(0.26)$ & $2(0.04)$ & $20(0.24)$ & $37(0.19)$ & 0.008 \\
\hline Collimator rotation & $23(0.40)$ & $11(0.19)$ & $14(0.17)$ & $48(0.25)$ & 0.016 \\
\hline Field size $X$ & $59(1.04)$ & $19(0.34)$ & $14(0.17)$ & $92(0.48)$ & $<0.001$ \\
\hline Field size $Y$ & $55(0.97)$ & $6(0.11)$ & $18(0.22)$ & $79(0.41)$ & $<0.001$ \\
\hline Treatment time $\%$ & $7(0.12)$ & $13(0.24)$ & $41(0.49)$ & $61(0.30)$ & $<0.001$ \\
\hline Wedge & $1(0.02)$ & $59(1.07)$ & $73(0.88)$ & $133(0.68)$ & $<0.001$ \\
\hline Applicator & - & - & $1(0.01)$ & 1 & - \\
\hline Energy & - & - & $26(0.31)$ & $26(0.13)$ & - \\
\hline Asymmetry & - & $13(0.24)$ & $13(0.16)$ & $26(0.13)$ & 0.002 \\
\hline Total & $160(2.81)$ & $123(2.23)$ & $220(2.65)$ & $503(2.58)$ & \\
\hline
\end{tabular}

${ }^{a}$ Results are expressed in terms of number $(\%)$ of treated fields with erroneous parameters over total number of treated fields. Comparison of error frequencies between treatment machines $(P$-value $) . *_{n}$, Number of treated fields.

therapy team but also valuable for research and management $[5,6,8-10]$.

The present study was designed to assess the frequency of errors during radiation treatment delivery that could be detected by the use of a RVS as well as the frequency of hypothetical errors related to the use of such verification systems.

Our main findings are that at least one setting was erroneous (true error) in $3.22 \%$ of the treated fields and that at least one deviation was found between the intended parameters and the data stored in the memory of the RVS computer (false error) for $1.17 \%$ of the fields.

In the present study, the parameters of interest included field size, gantry angle, collimator angle, treatment time, choice of energy, use of accessories such as wedges or blocking trays applicator and symmetry. Couch settings were not examined. Hence, the actual number of true errors that occur during daily treatment delivery is probably underestimated. However, our data clearly demonstrate that a large number of errors can be detected and prevented by using RVS.

Substantial variability exists between previous studies reporting frequencies of errors observed during treatment delivery. Frequencies ranging from 0.6 to $14 \%$ of the fields containing at least one error have been reported [2,3,11-16]. Such discrepancies can be explained by methodological

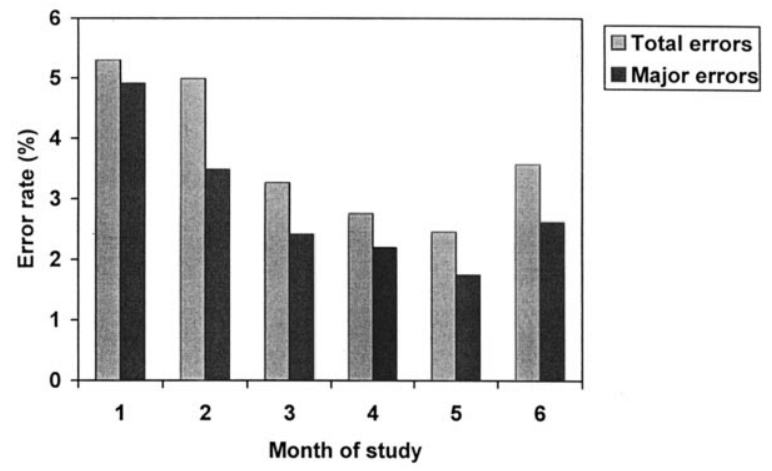

Fig. 1. Percentage of total and major errors per month. differences. These include variations in the treatment parameters examined, in the treatment machines used, in the tolerance tables and/or in the technique of errors detection. Some studies use voluntary reports based on 'subjective' manual methods whereas others use more sophisticated techniques based on 'objective' computerized recordings. We believe that the error frequencies reported in this paper are fairly accurate because (i) most settings were controlled, (ii) the intended parameters and the actually used settings were compared by using a computer, and (iii) the study was carried out over a long period of time making improved alertness of the radiation nurses or of radiographers unlikely. The high frequency of major errors is related to the definition of major errors.

For some parameters, the frequency of errors was significantly different between the three treatment machines. More errors in field size and collimator rotation occurred when the Cobalt unit was used. This is most likely related to the type of display; these two parameters being analogic on the Alcyon, whereas digital displays are present on the linacs. The gantry angle was set erroneously less frequently on the Orion than on the Saturne or the Alcyon. This is best explained by the blocking trays used on the Orion in our department. This system can be set only when the gantry angle is at 0 or $180^{\circ}$. More deviations in the setting of gantry or collimator rotation angles occurred when these parameters were planned to be different from 0, 90, 180 and $270^{\circ}$. This finding suggests that the use of RVS would be

Table 6

Number $(\%)$ of false errors (see text for definition) according to parameters for the 1885 fields recorded in the RVS

\begin{tabular}{lr}
\hline Parameter & \multicolumn{1}{l}{ Errors } \\
\hline Gantry & $3(0.16)$ \\
Collimator rotation & $6(0.32)$ \\
Field size $Y$ and/or $X$ & $9(0.48)$ \\
Treatment time & $1(0.05)$ \\
Wedge & $3(0.16)$ \\
Total & $22(1.17)$
\end{tabular}


especially valuable for radiation treatment using complex multiple beams with diverse accessories according to $3 \mathrm{D}$ treatment planning [7].

The higher frequency of errors at the beginning of the study period may have at least two explanations. On the one hand, using the RVS, radiation nurses or radiographers are confident in the system and their capacity to control their own parameters adjustments is reduced. After discontinuing the use of RVS, time is necessary to recover adequate watchfulness [4]. On the other hand, several recently certified radiation nurses or radiographers began to work in the department just before the start of the study. Their limited experience may have contributed to the higher frequency of errors observed in the early weeks of the study.

The frequency of false errors indicates that the use of a record and verifiy system could be associated with input errors into the system computer. Hence, if erroneous data introduced in the computer are used for treatment setting, the RVS (initially designed to prevent accidental errors) could lead to the introduction of errors that would be repeated throughout the treatment delivery. However, if the first treatment setting is performed according to the data recorded in the patient treatment chart, any input error in the RVS would be detected as any discrepancy between set parameters and parameters stored in the computer memory will inhibit the irradiation beam. Hence, input errors could be corrected and would not alter the treatment actually delivered. It is therefore of paramount importance to verify the entire treatment setting with a parallel procedure before and during the first treatment session $[6,9,13]$. Moreover, the automatic transfer of all treatment parameters from the simulator and the treatment planning system to the RVS and the treatment room should also reduce the risk of input errors.

The present study clearly demonstrates that accidental deviations from the prescribed treatment parameters occur during radiation treatment. Computer monitoring and verification of daily settings play significant roles in modern radiotherapy to achieve the desired degree of precision. However, correct input of treatment parameters into the computer is critical because these data will be used for the entire treatment period. Indeed, excessive confidence in the RVS could lead to errors that would be repeated throughout the treatment delivery. Therefore, it is of utmost importance to verify the entire treatment setting at the first treatment session.

\section{Acknowledgements}

The authors like to acknowledge the conscientious work and dedication of the members of the clinical and medical computing teams whose efforts are analyzed here. They are also indebted to GE Medical Systems for preparing a 'blind' version of the RVS software.

\section{References}

[1] Dutreix A. When and how can we improve precision in radiotherapy? Radiother. Oncol. 1984;2:275-292.

[2] Fraass BA, Lash KL, Matrone GM, et al. The impact of treatment complexity and computer-control delivery technology on treatment delivery errors. Int. J. Radiat. Oncol. Biol. Phys. 1998;42:651-659.

[3] Fredrickson DH, Karzmark CJ, Rust DC, Tuschman M. Experience with computer monitoring, verification and record keeping in radiotherapy procedures using a clinac-4. Int. J. Radiat. Oncol. Biol. Phys. 1979;5:415-418.

[4] Green R. The psychology of human error. In: Atkinson RS, Adams AP, editors. Recent advances in anaesthesia and analgesia, Edimburgh: Churchill Livingstone, 1995. pp. 1-15.

[5] ICRU 24. International Commission on Radiation Units and Measurements (ICRU), Prescribing, Recording, and Reporting Photon Beam Therapy. ICRU Report 50. Bethesda, Maryland, 1993.

[6] Kartha PKI, Chung-Bin A, Wachtor T, Hendrickson FR. Accuracy in radiotherapy treatment. Int. J. Radiat. Oncol. Biol. Phys. 1977;2:797799.

[7] Klein EE, Drzymala RE, Williams R, Westfall LA, Purdy JA. A change in treatment process with a modern record and verify system. Int. J. Radiat. Oncol. Biol. Phys. 1998;42:1163-1168.

[8] Leunens G, Verstraete J, Van den Bogaert W, Van Dam J, Dutreix A, van der Schueren E. Human errors in data transfer during the preparation and delivery of radiation treatment affecting the final result: 'garbage in, garbage out'. Radiother. Oncol. 1992;23:217-222.

[9] Macklis RM, Meier T, Weinhous MS. Error rates in clinical radiotherapy. J. Clin. Oncol. 1998;16:551-556.

[10] Mohan R, Caley R. Standardization of therapy machine interface for treatment monitoring. Int. J. Radiat. Oncol. Biol. Phys. 1983;9:12251229.

[11] Mohan R, Podmaniczky KC, Caley R, Lapidus A, Laughlin JS. A computerized record and verify system for radiation treatments. Int. J. Radiat. Oncol. Biol. Phys. 1984;10:1975-1985.

[12] Muller-Runkel R, Watkins SS. Introducing a computerized record and verify system: its impact on the reduction of treatment errors. Med. Dosim. 1991;16:19-22.

[13] Perez CA, Gardner P, Glasgow GP. Radiotherapy quality assurance in clinical trials. Int. J. Radiat. Oncol. Biol. Phys. 1984;10(Suppl. 1):119-125.

[14] Podmaniczky KC, Mohan R, Kutcher GJ, Kestler C, Vikram B. Clinical experience with a computerized record and verify system. Int. J. Radiat. Oncol. Biol. Phys. 1985;11:1529-1537.

[15] Rosenbloom ME, Killick LJ, Bentley RE. Verification and recording of radiotherapy treatments using a small computer. Br. J. Radiol. 1977;50:637-644.

[16] Valli MC, Prina M, Bossi A, et al. Evaluation of most frequent errors in daily compilation and use of a radiation treatment chart. Radiother. Oncol. 1994;32:87-89. 